\title{
Unified Bernstein and Bleimann-Butzer-Hahn basis and its properties
}

\author{
Mehmet Ali Özarslan and Mehmet Bozer*
}

"Correspondence:

mehmet.bozer@emu.edu.tr

Eastern Mediterranean University,

Mersin 10, Gazimagusa, TRNC,

Turkey

\begin{abstract}
In this paper we introduce the unification of Bernstein and Bleimann-Butzer-Hahn basis via the generating function. We give the representation of this unified family in terms of Apostol-type polynomials and Stirling numbers of the second kind. More generating functions of trigonometric type are also obtained to this unification.

MSC: 11B65; 11B68; 41A10; 30C15

Keywords: generating function; Bernstein polynomials; Bernoulli polynomials; Euler polynomials; Genocchi polynomials; Stirling numbers of the second kind
\end{abstract}

\section{Introduction}

In this paper, we introduce a two-parameter generating function, which generates not only the Bernstein basis polynomials, but also the Bleimann-Butzer-Hahn basis functions. The generating function that we propose is given by

$$
\mathcal{G}_{a, b}(t, x ; k, m):=\left[\frac{2^{1-k} x^{k} t^{k}}{(1+a x)^{k}}\right]^{m} \frac{1}{(m k) !} e^{t\left[\frac{1+b x}{1+a x}\right]}=\sum_{n=0}^{\infty} \mathcal{P}_{n}^{(a, b)}(x ; k, m) \frac{t^{n}}{n !}
$$

where $k, m \in \mathbb{Z}^{+}:=\{1,2, \ldots\}, a, b \in \mathbb{R}, t \in \mathbb{C}$. Here, $x \in I$ where $I$ is a subinterval of $\mathbb{R}$ such that the expansion in (1) is valid. The following two cases will be important for us.

1 . The case $a=0, b=-1$. In this case, we let $x \in[0,1]$ and we see that

$$
\mathcal{G}_{0,-1}(t, x ; k, m)=\left[2^{1-k} x^{k} t^{k}\right]^{m} \frac{1}{(m k) !} e^{t[1-x]}=\sum_{n=0}^{\infty} \mathcal{P}_{n}^{(0,-1)}(x ; k, m) \frac{t^{n}}{n !}
$$

generates the unifying Bernstein basis polynomials $\mathcal{P}_{n}^{(0,-1)}(x ; k, m):=\mathcal{B}_{n}(m k, x)$ which were introduced and investigated in [1]. We should note further that $\mathcal{G}_{0,-1}(t, x ; 1, m)$ gives

$$
\mathcal{G}_{0,-1}(t, x ; 1, m)=[x t]^{m} \frac{1}{m !} e^{t[1-x]}=\sum_{n=0}^{\infty} \mathcal{B}_{n}(m, x) \frac{t^{n}}{n !}
$$

which generates the celebrated Bernstein basis polynomials (see [2-8])

$$
\mathcal{B}_{n}(m, x):=B_{m}^{n}(x)=\left(\begin{array}{c}
n \\
m
\end{array}\right) x^{k}(1-x)^{n-m} .
$$

๑) 2013 Özarslan and Bozer; licensee Springer. This is an Open Access article distributed under the terms of the Creative Commons Attribution License (http://creativecommons.org/licenses/by/2.0), which permits unrestricted use, distribution, and reproduction in any medium, provided the original work is properly cited. 
Note that the Bernstein operators $B_{n}: C[0,1] \rightarrow C[0,1]$ are given by

$$
B_{n}(f ; x)=\sum_{m=0}^{n} f\left(\frac{m}{n}\right)\left(\begin{array}{c}
n \\
m
\end{array}\right) x^{k}(1-x)^{n-m}, \quad n \in \mathbb{N}:=\{1,2, \ldots\}
$$

and by the Korovkin theorem, it is known that $B_{n}(f ; x) \rightrightarrows f(x)$ for all $f \in C[0,1]$, where $C[0,1]$ denotes the space of continuous functions defined on $[0,1]$, and the notation ' $\rightrightarrows$ ' denotes the uniform convergence with respect to the usual supremum norm on $C[0,1]$. Very recently, interesting properties of Bernstein polynomials were discussed in [7, 9-11] and [12].

2. The case $a=1, b=0$. In this case, we let $x \in[0, \infty)$ and define

$$
\begin{aligned}
\mathcal{G}_{1,0}(t, x ; k, m) & :=\left[\frac{2^{1-k} x^{k} t^{k}}{(1+x)^{k}}\right]^{m} \frac{1}{(m k) !} e^{t\left[\frac{1}{1+x}\right]} \\
& =\sum_{n=0}^{\infty} \mathcal{P}_{n}^{(1,0)}(x ; k, m) \frac{t^{n}}{n !} .
\end{aligned}
$$

We will see that this generating function produces the generalized Bleimann-Butzer-Hahn basis functions $\mathcal{P}_{n}^{(1,0)}(x ; k, m):=\mathcal{H}_{n}(m k, x)$. Furthermore, the special case

$$
\begin{aligned}
\mathcal{G}_{1,0}(t, x ; 1, m) & =\left[\frac{x t}{(1+x)}\right]^{m} \frac{1}{(m k) !} e^{t\left[\frac{1}{1+x}\right]} \\
& =\sum_{n=0}^{\infty} \mathcal{H}_{n}(m, x) \frac{t^{n}}{n !}
\end{aligned}
$$

generates the well-known Bleimann-Butzer-Hahn basis functions:

$$
\mathcal{H}_{n}(m, x):=H_{m}^{n}(x)=\left(\begin{array}{c}
n \\
m
\end{array}\right) \frac{x^{m}}{(1+x)^{n}}
$$

The Bleimann-Butzer-Hahn operators were introduced in [5] and defined by

$$
L_{n}(f ; x)=\frac{1}{(1+x)^{n}} \sum_{m=0}^{n} f\left(\frac{m}{n}\right)\left(\begin{array}{c}
n \\
m
\end{array}\right) x^{m} ; \quad x \in[0, \infty), n \in \mathbb{N} .
$$

Denoting $C_{B}[0, \infty)$ by the space of real-valued bounded continuous functions defined on $[0, \infty)$, they proved that $L_{n}(f) \rightarrow f$ as $n \rightarrow \infty$. On the other hand, the convergence is uniform on each compact subset of $[0, \infty)$, where the norm is the usual supremum norm of $C_{B}[0, \infty)$. For the review of the results concerning the Bleimann-Butzer-Hahn operators obtained in the period 1980-2009, we refer to [13].

The following theorem gives the explicit representation of the basis family defined in (1). Note that throughout the paper, we let $\mathcal{P}_{n}^{(a, b)}(x ; k, m):=0$ for $n \leq m k$.

Theorem 1 If $n \geq m k$, we have

$$
\mathcal{P}_{n}^{(a, b)}(x ; k, m)=2^{(1-k) m} x^{m k}\left(\begin{array}{c}
n \\
m k
\end{array}\right) \frac{(1+b x)^{n-m k}}{(1+a x)^{n}}
$$


Proof Direct calculations give

$$
\begin{aligned}
\mathcal{G}_{a, b}(t, x ; k, m) & =\left[\frac{2^{1-k} x^{k} t^{k}}{(1+a x)^{k}}\right]^{m} \frac{1}{(m k) !} e^{t\left[\frac{1+b x}{1+a x}\right]} \\
& =\frac{2^{(1-k) m}}{(m k) !}\left(\frac{x t}{1+a x}\right)^{m k} \sum_{n=0}^{\infty}\left(\frac{1+b x}{1+a x}\right)^{n} \frac{t^{n}}{n !} \\
& =2^{(1-k) m} x^{m k} \sum_{n=m k}^{\infty}\left(\begin{array}{c}
n \\
m k
\end{array}\right) \frac{(1+b x)^{n-m k}}{(1+a x)^{n}} \frac{t^{n}}{n !} .
\end{aligned}
$$

Comparing (1) and (2), we get the result.

Corollary 2 By taking $a=0, b=-1$ in Theorem 1 , we obtain the explicit representation of the unifying Bernstein basis polynomials [1]:

$$
\mathcal{P}_{n}^{(0,-1)}(x ; k, m):=\mathcal{B}_{n}(m k, x)=2^{(1-k) m} x^{m k}\left(\begin{array}{c}
n \\
m k
\end{array}\right)(1-x)^{n-m k} .
$$

Furthermore, $\mathcal{B}_{n}(m, x)=B_{m}^{n}(x)$ is the well-known Bernstein basis.

Corollary 3 Taking $a=1, b=0$ in Theorem 1, we get the explicit representation of the generalized Bleimann-Butzer-Hahn basis:

$$
\mathcal{P}_{n}^{(1,0)}(x ; k, m):=\mathcal{H}_{n}(m k, x)=2^{(1-k) m} x^{m k}\left(\begin{array}{c}
n \\
m k
\end{array}\right) \frac{1}{(1+x)^{n}}
$$

Moreover, $\mathcal{H}_{n}(m, x)=H_{m}^{n}(x)$ is the Bleimann-Butzer-Hahn basis function.

We organize the paper as follows. In Section 2, we obtain the representation of this unified family in terms of Apostol-type polynomials and Stirling numbers of the second kind. In Section 3, we give more trigonometric generating functions for this unification and obtain a certain summation formula. All the special cases are listed at the end of each theorem.

\section{Representation in terms of Apostol-type polynomials and Stirling numbers}

Recently [14], the first author introduced the unification of the Apostol-Bernoulli, Euler and Genocchi polynomials by

$$
\begin{gathered}
\mathcal{P}_{a, b}^{(\alpha)}(x ; t ; k, \beta):=\left(\frac{2^{1-k} t^{k}}{\beta^{b} e^{t}-a^{b}}\right)^{\alpha} e^{x t}=\sum_{n=0}^{\infty} Q_{n, \beta}^{(\alpha)}(x ; k, a, b) \frac{t^{n}}{n !} \\
\left(k \in \mathbb{N}_{0} ; a, b \in \mathbb{R} \backslash\{0\} ; \alpha, \beta \in \mathbb{C}\right) .
\end{gathered}
$$

For the convergence of the series in (3), we refer to [14, p.2453].

Some of the well-known polynomials included by $Q_{n, \beta}^{(\alpha)}(x ; k, a, b)$ are listed below.

Remark 4 Having $k=a=b=1$ and $\beta=\lambda$ in (3), we get

$$
Q_{n, \lambda}^{(\alpha)}(x ; 1,1,1)=\mathcal{B}_{n}^{(\alpha)}(x ; \lambda)
$$


Note that $\mathcal{B}_{n}^{(\alpha)}(x ; \lambda)$ are the generalized Apostol-Bernoulli polynomials defined through the following generating relation:

$$
\begin{aligned}
& \left(\frac{t}{\lambda e^{t}-1}\right)^{\alpha} e^{x t}=\sum_{n=0}^{\infty} \mathcal{B}_{n}^{(\alpha)}(x ; \lambda) \frac{t^{n}}{n !} \\
& (|t|<2 \pi \text { when } \lambda=1 ;|t|<|\log \lambda| \text { when } \lambda \neq 1),
\end{aligned}
$$

where $\alpha$ and $\lambda$ are arbitrary real or complex parameters and $x \in \mathbb{R}$. Note that when $\lambda \neq 1$, the order $\alpha$ should be restricted to nonnegative integer values. These polynomials were introduced by Luo and Srivastava [15] and investigated in [16, 17] and [18]. The ApostolBernoulli polynomials and numbers are obtained by the generalized Apostol-Bernoulli polynomials, respectively, as follows:

$$
B_{n}(x ; \lambda)=\mathcal{B}_{n}^{(1)}(x ; \lambda), \quad B_{n}(\lambda)=B_{n}(0 ; \lambda) \quad\left(n \in \mathbb{N}_{0}\right) .
$$

Taking $\lambda=1$ in the above relations, we obtain the classical Bernoulli polynomials $B_{n}(x)$ and Bernoulli numbers $B_{n}$.

Remark 5 Letting $k=-2 a=b=1$ and $2 \beta=\lambda$ in (3), we get

$$
Q_{n, \frac{\lambda}{2}}^{(\alpha)}\left(x ; 1, \frac{-1}{2}, 1\right)=\mathcal{G}_{n}^{\alpha}(x ; \lambda),
$$

the Apostol-Genocchi polynomial of order $\alpha$ (arbitrary real or complex) which was defined by $[19,20]$. Here the parameter $\lambda$ is arbitrary real or complex. These polynomials are given as follows:

$$
\begin{aligned}
& \left(\frac{2 t}{\lambda e^{t}+1}\right)^{\alpha} e^{x t}=\sum_{n=0}^{\infty} \mathcal{G}_{n}^{\alpha}(x ; \lambda) \frac{t^{n}}{n !} \\
& (|t|<\pi \text { when } \lambda=1 ;|t|<|\log (-\lambda)| \text { when } \lambda \neq 1) .
\end{aligned}
$$

Note that when $\lambda \neq-1$, the order $\alpha$ should be restricted to nonnegative integer values. The Apostol-Genocchi polynomials and numbers are respectively given by

$$
G_{n}(x ; \lambda)=\mathcal{G}_{n}^{1}(x ; \lambda), \quad G_{n}(\lambda)=G_{n}(0 ; \lambda) .
$$

When $\lambda=1$, the above relations give the classical Genocchi polynomials $G_{n}(x)$ and Genocchi numbers $G_{n}$.

Although our results do not contain the Apostol-Euler polynomials, for the sake of completeness, we give their definitions as a special case of the polynomial family $Q_{n, \beta}^{(\alpha)}(x ; k, a, b)$.

Remark 6 Setting $k+1=-a=b=1$ and $\beta=\lambda$ in (3), we get

$$
Q_{n, \lambda}^{(\alpha)}(x ; 0,-1,1)=\mathcal{E}_{n}^{(\alpha)}(x ; \lambda)
$$


Recall that the Apostol-Euler polynomials $\mathcal{E}_{n}^{(\alpha)}(x ; \lambda)$ are generalized by Luo [21] and given by the generating relation

$$
\begin{aligned}
& \left(\frac{2}{\lambda e^{t}+1}\right)^{\alpha} e^{x t}=\sum_{n=0}^{\infty} \mathcal{E}_{n}^{\alpha}(x ; \lambda) \frac{t^{n}}{n !} \\
& \quad\left(|t|<\pi \text { when } \lambda=1 ;|t|<|\log (-\lambda)| \text { when } \lambda \neq 1 ; 1^{\alpha}:=1\right)
\end{aligned}
$$

for arbitrary real or complex parameters $\alpha$ and $\lambda$ and $x \in \mathbb{R}$. The Apostol-Euler polynomials and numbers are given respectively by

$$
E_{n}(x ; \lambda)=\mathcal{E}_{n}^{1}(x ; \lambda), \quad E_{n}(\lambda)=E_{n}(1 ; \lambda) .
$$

When $\lambda=1$, the above relations give the classical Euler polynomials $E_{n}(x)$ and Euler numbers $E_{n}$.

Now, recall that the Stirling numbers of the second kind are denoted by $S(j, i)$ and defined by (see [22, p.58 (15)])

$$
\left(e^{t}-1\right)^{i}=i ! \sum_{j=i}^{\infty} S(j, i) \frac{t^{j}}{j !}
$$

The following theorem states an interesting explicit representation of the unified basis in terms of Apostol-type polynomials and relation between Stirling numbers of the second kind.

Theorem 7 The following representation:

$$
\begin{aligned}
\mathcal{P}_{n}^{(a, b)}(x ; k, m)= & \frac{1}{(m k) !}\left(\frac{x}{1+a x}\right)^{m k} \sum_{i=0}^{m}\left(\begin{array}{c}
m \\
i
\end{array}\right)\left(\beta^{d}-c^{d}\right)^{m-i} \beta^{i d} i ! \\
& \times \sum_{j=i}^{n}\left(\begin{array}{c}
n \\
j
\end{array}\right) S(j, i) Q_{n-j, \beta}^{(m)}\left(\frac{1+b x}{1+a x} ; k, c, d\right)
\end{aligned}
$$

holds true between the unified Bernstein and Bleimann-Butzer-Hahn basis and Apostoltype polynomials.

Proof We get, using (1), that

$$
\begin{aligned}
\sum_{n=0}^{\infty} & \mathcal{P}_{n}^{(a, b)}(x ; k, m) \frac{t^{n}}{n !} \\
& =\mathcal{G}_{a, b}(t, x ; k, m) \\
& =\left[\frac{2^{1-k} x^{k} t^{k}}{(1+a x)^{k}}\right]^{m} \frac{1}{(m k) !} e^{t\left[\frac{1+b x}{1+a x}\right]} \\
& =\frac{1}{(m k) !}\left(\frac{x}{1+a x}\right)^{m k}\left[\frac{2^{1-k} t^{k}}{\beta^{d} e^{t}-c^{d}}\right]^{m} e^{t\left[\frac{1+b x}{1+a x}\right]}\left(\beta^{d} e^{t}-c^{d}\right)^{m} \\
& =\frac{1}{(m k) !}\left(\frac{x}{1+a x}\right)^{m k}\left[\frac{2^{1-k} t^{k}}{\beta^{d} e^{t}-c^{d}}\right]^{m} e^{t\left[\frac{1+b x}{1+a x}\right]}\left(\beta^{d}-c^{d}+\beta^{d}\left[e^{t}-1\right]\right)^{m} .
\end{aligned}
$$


On the other hand, since

$$
\begin{aligned}
\left(\beta^{d}-c^{d}+\beta^{d}\left[e^{t}-1\right]\right)^{m} & =\sum_{i=0}^{m}\left(\begin{array}{c}
m \\
i
\end{array}\right)\left(\beta^{d}-c^{d}\right)^{m-i} \beta^{i d}\left[e^{t}-1\right]^{i} \\
& =\sum_{i=0}^{m}\left(\begin{array}{c}
m \\
i
\end{array}\right)\left(\beta^{d}-c^{d}\right)^{m-i} \beta^{i d} i ! \sum_{j=i}^{\infty} S(j, i) \frac{t^{j}}{j !},
\end{aligned}
$$

we can write from (4) that

$$
\begin{aligned}
\sum_{n=0}^{\infty} \mathcal{P}_{n}^{(a, b)}(x ; k, m) \frac{t^{n}}{n !} \\
=\frac{1}{(m k) !}\left(\frac{x}{1+a x}\right)^{m k}\left[\frac{2^{1-k} t^{k}}{\beta^{b} e^{t}-a^{b}}\right]^{m} e^{t\left[\frac{1+b x}{1+a x}\right]} \\
\quad \times \sum_{i=0}^{m}\left(\begin{array}{c}
m \\
i
\end{array}\right)\left(\beta^{b}-a^{b}\right)^{m-i} \beta^{i b} i ! \sum_{j=i}^{\infty} S(j, i) \frac{t^{j}}{j !} .
\end{aligned}
$$

Now, using (3) in the above relation, we get

$$
\begin{aligned}
\sum_{n=0}^{\infty} \mathcal{P}_{n}^{(a, b)}(x ; k, m) \frac{t^{n}}{n !} & \frac{1}{(m k) !}\left(\frac{x}{1+a x}\right)^{m k} \sum_{n=0}^{\infty} Q_{n, \beta}^{(m)}\left(\frac{1+b x}{1+a x} ; k, c, d\right) \frac{t^{n}}{n !} \\
& \times \sum_{i=0}^{m}\left(\begin{array}{c}
m \\
i
\end{array}\right)\left(\beta^{d}-c^{d}\right)^{m-i} \beta^{i d} i ! \sum_{j=i}^{\infty} S(j, i) \frac{t^{j}}{j !} \\
= & \frac{1}{(m k) !}\left(\frac{x}{1+a x}\right)^{m k} \sum_{n=0}^{\infty}\left\{\sum_{i=0}^{m}\left(\begin{array}{c}
m \\
i
\end{array}\right)\left(\beta^{d}-c^{d}\right)^{m-i} \beta^{i d} i !\right. \\
& \left.\times \sum_{j=i}^{n}\left(\begin{array}{c}
n \\
j
\end{array}\right) S(j, i) Q_{n-j, \beta}^{(m)}\left(\frac{1+b x}{1+a x} ; k, c, d\right)\right\} \frac{t^{n}}{n !} .
\end{aligned}
$$

Whence the result.

Now, we list some important corollaries of the above theorem.

Corollary 8 Since $\mathcal{P}_{n}^{(0,-1)}(x ; 1, m)=B_{m}^{n}(x)$ and $Q_{n, \lambda}^{(\alpha)}(x ; 1,1,1)=\mathcal{B}_{n}^{(\alpha)}(x ; \lambda)$, we obtain the following [1]:

$$
B_{m}^{n}(x)=\frac{x^{m}}{m !} \sum_{i=0}^{m}\left(\begin{array}{c}
m \\
i
\end{array}\right)(\lambda-1)^{m-i} \lambda^{i} i ! \sum_{j=i}^{n}\left(\begin{array}{c}
n \\
j
\end{array}\right) S(j, i) \mathcal{B}_{n-j}^{(m)}(1-x ; \lambda) .
$$

Furthermore, for $\lambda=1$, we have the following known relation:

$$
B_{m}^{n}(x)=x^{m} \sum_{j=m}^{n}\left(\begin{array}{l}
n \\
j
\end{array}\right) S(j, m) B_{n-j}^{(m)}(1-x) .
$$


Corollary 9 Since $\mathcal{P}_{n}^{(0,-1)}(x ; 1, m)=B_{m}^{n}(x)$ and $Q_{n, \frac{\lambda}{2}}^{(\alpha)}\left(x ; 1, \frac{-1}{2}, 1\right)=\mathcal{G}_{n}^{\alpha}(x ; \lambda)$, we get

$$
B_{m}^{n}(x)=\frac{x^{m}}{2^{m} m !} \sum_{i=0}^{m}\left(\begin{array}{c}
m \\
i
\end{array}\right)(\lambda+1)^{m-i} \lambda^{i} i ! \sum_{j=i}^{n}\left(\begin{array}{l}
n \\
j
\end{array}\right) S(j, i) \mathcal{G}_{n-j}^{m}(1-x ; \lambda) .
$$

Corollary 10 Since $\mathcal{P}_{n}^{(1,0)}(x ; 1, m)=H_{m}^{n}(x)$ and $Q_{n, \lambda}^{(\alpha)}(x ; 1,1,1)=\mathcal{B}_{n}^{(\alpha)}(x ; \lambda)$, we obtain

$$
\begin{aligned}
H_{m}^{n}(x)= & \frac{1}{m !}\left(\frac{x}{1+x}\right)^{m} \sum_{i=0}^{m}\left(\begin{array}{c}
m \\
i
\end{array}\right)(\lambda-1)^{m-i} \lambda^{i} i ! \\
& \times \sum_{j=i}^{n}\left(\begin{array}{c}
n \\
j
\end{array}\right) S(j, i) \mathcal{B}_{n-j}^{(m)}\left(\frac{1}{1+x} ; \lambda\right) .
\end{aligned}
$$

Furthermore, when $\lambda=1$, we have the following:

$$
H_{m}^{n}(x)=\left(\frac{x}{1+x}\right)^{m} \sum_{j=m}^{n}\left(\begin{array}{l}
n \\
j
\end{array}\right) S(j, m) B_{n-j}^{(m)}\left(\frac{1}{1+x}\right)
$$

Corollary 11 Since $\mathcal{P}_{n}^{(1,0)}(x ; 1, m)=H_{m}^{n}(x)$ and $Q_{n, \frac{\lambda}{2}}^{(\alpha)}\left(x ; 1, \frac{-1}{2}, 1\right)=\mathcal{G}_{n}^{\alpha}(x ; \lambda)$, we get

$$
\begin{aligned}
H_{m}^{n}(x)= & \frac{1}{2^{m} m !}\left(\frac{x}{1+x}\right)^{m} \sum_{i=0}^{m}\left(\begin{array}{c}
m \\
i
\end{array}\right)(\lambda-1)^{m-i} \lambda^{i} i ! \\
& \times \sum_{j=i}^{n}\left(\begin{array}{c}
n \\
j
\end{array}\right) S(j, i) \mathcal{G}_{n-j}^{m}\left(\frac{1}{1+x} ; \lambda\right) .
\end{aligned}
$$

\section{Generating functions of trigonometric type}

In this section, we obtain a trigonometric generating relation for the unified Bernstein and Bleimann-Butzer-Hahn basis. Furthermore, we give a certain summation formula for this unification. We start with the following theorem.

Theorem 12 For the unified family, we have the following implicit summation formulae:

$$
\begin{aligned}
& {\left[\frac{2^{1-2 l} x^{2 l}}{(1+a x)^{2 l}}\right]^{m} \frac{\left(-t^{2}\right)^{l m}}{(2 l m) !} \cos t\left(\frac{1+b x}{1+a x}\right)=\sum_{n=0}^{\infty}(-1)^{n} \mathcal{P}_{2 n}^{(a, b)}(x ; 2 l, m) \frac{t^{2 n}}{(2 n) !}} \\
& {\left[\frac{2^{1-2 l} x^{2 l}}{(1+a x)^{2 l}}\right]^{m} \frac{\left(-t^{2}\right)^{l m}}{(2 l m) !} \sin t\left(\frac{1+b x}{1+a x}\right)=\sum_{n=0}^{\infty}(-1)^{n} \mathcal{P}_{2 n+1}^{(a, b)}(x ; 2 l, m) \frac{t^{2 n+1}}{(2 n+1) !}}
\end{aligned}
$$

and

$$
\begin{aligned}
& {\left[\frac{2^{-2 l} x^{2 l+1}}{(1+a x)^{2 l+1}}\right]^{2 j} \frac{\left(-t^{2}\right)^{(2 l+1) j}}{(2 j(2 l+1)) !} \cos t\left(\frac{1+b x}{1+a x}\right)=\sum_{n=0}^{\infty}(-1)^{n} \mathcal{P}_{2 n}^{(a, b)}(x ; 2 l+1,2 j) \frac{t^{2 n}}{(2 n) !}} \\
& {\left[\frac{2^{-2 l} x^{2 l+1}}{(1+a x)^{2 l+1}}\right]^{2 j} \frac{\left(-t^{2}\right)^{(2 l+1) j}}{(2 j(2 l+1)) !} \sin t\left(\frac{1+b x}{1+a x}\right)=\sum_{n=0}^{\infty}(-1)^{n} \mathcal{P}_{2 n+1}^{(a, b)}(x ; 2 l+1,2 j) \frac{t^{2 n+1}}{(2 n+1) !}}
\end{aligned}
$$


Finally,

$$
\begin{aligned}
& {\left[\frac{2^{-2 l} x^{2 l+1}}{(1+a x)^{2 l+1}}\right]^{2 j+1} \frac{\left(-t^{2}\right)^{(2 l j+l+j)}}{[(2 j+1)(2 l+1)] !} t \sin t\left(\frac{1+b x}{1+a x}\right)} \\
& \quad=\sum_{n=0}^{\infty}(-1)^{n} \mathcal{P}_{2 n}^{(a, b)}(x ; 2 l+1,2 j+1) \frac{t^{2 n}}{(2 n) !}, \\
& {\left[\frac{2^{-2 l} x^{2 l+1}}{(1+a x)^{2 l+1}}\right]^{2 j+1} \frac{\left(-t^{2}\right)^{(2 l j+l+j)}}{[(2 j+1)(2 l+1)] !} t \cos t\left(\frac{1+b x}{1+a x}\right)} \\
& \quad=\sum_{n=0}^{\infty}(-1)^{n} \mathcal{P}_{2 n+1}^{(a, b)}(x ; 2 l+1,2 j+1) \frac{t^{2 n+1}}{(2 n+1) !} .
\end{aligned}
$$

Proof Writing $k=2 l\left(l \in \mathbb{N}_{0}\right)$ in (1), we get

$$
\left[\frac{2^{1-2 l} x^{2 l} t^{2 l}}{(1+a x)^{2 l}}\right]^{m} \frac{1}{(2 l m) !} e^{t\left[\frac{1+b x}{1+a x}\right]}=\sum_{n=0}^{\infty} \mathcal{P}_{n}^{(a, b)}(x ; 2 l, m) \frac{t^{n}}{n !} .
$$

Letting $t \rightarrow i t$, we get

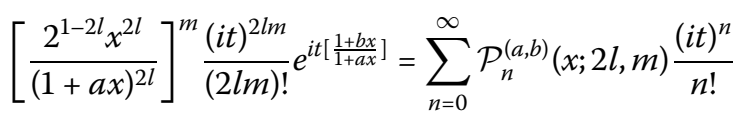

and hence

$$
\begin{aligned}
& {\left[\frac{2^{1-2 l} x^{2 l}}{(1+a x)^{2 l}}\right]^{m} \frac{\left(-t^{2}\right)^{l m}}{(2 l m) !}\left\{\cos t\left(\frac{1+b x}{1+a x}\right)+i \sin t\left(\frac{1+b x}{1+a x}\right)\right\}} \\
& =\sum_{n=0}^{\infty} \mathcal{P}_{2 n}^{(a, b)}(x ; 2 l, m) \frac{(i t)^{2 n}}{(2 n) !}+\sum_{n=0}^{\infty} \mathcal{P}_{2 n+1}^{(a, b)}(x ; 2 l, m) \frac{(i t)^{2 n+1}}{(2 n+1) !} \\
& =\sum_{n=0}^{\infty}(-1)^{n} \mathcal{P}_{2 n}^{(a, b)}(x ; 2 l, m) \frac{t^{2 n}}{(2 n) !} \\
& \quad+i \sum_{n=0}^{\infty}(-1)^{n} \mathcal{P}_{2 n+1}^{(a, b)}(x ; 2 l, m) \frac{t^{2 n+1}}{(2 n+1) !}
\end{aligned}
$$

Equating real and imaginary parts, we get (5).

Now, taking $k=2 l+1$ and $m=2 j\left(l, j \in \mathbb{N}_{0}\right)$ in (1), we obtain

$$
\left[\frac{2^{1-(2 l+1)} x^{2 l+1} t^{2 l+1}}{(1+a x)^{2 l+1}}\right]^{2 j} \frac{1}{(2 j(2 l+1)) !} e^{t[1+b x}\left[\begin{array}{l}
1+a x \\
\text { a }
\end{array}=\sum_{n=0}^{\infty} \mathcal{P}_{n}^{(a, b)}(x ; 2 l+1,2 j) \frac{t^{n}}{n !} .\right.
$$

Putting $t \rightarrow i t$,

$$
\left[\frac{2^{-2 l} x^{2 l+1}(i t)^{2 l+1}}{(1+a x)^{2 l+1}}\right]^{2 j} \frac{1}{(2 j(2 l+1)) !} e^{i t\left[\frac{1+b x}{1+a x}\right]}=\sum_{n=0}^{\infty} \mathcal{P}_{n}^{(a, b)}(x ; 2 l+1,2 j) \frac{(i t)^{n}}{n !} .
$$


Therefore, we get

$$
\begin{aligned}
& {\left[\frac{2^{-2 l} x^{2 l+1}}{(1+a x)^{2 l+1}}\right]^{2 j} \frac{\left(-t^{2}\right)^{(2 l+1) j}}{(2 j(2 l+1)) !}\left\{\cos t\left(\frac{1+b x}{1+a x}\right)+i \sin t\left(\frac{1+b x}{1+a x}\right)\right\}} \\
& \quad=\sum_{n=0}^{\infty}(-1)^{n} \mathcal{P}_{2 n}^{(a, b)}(x ; 2 l+1,2 j) \frac{t^{2 n}}{(2 n) !}+i \sum_{n=0}^{\infty}(-1)^{n} \mathcal{P}_{2 n+1}^{(a, b)}(x ; 2 l+1,2 j) \frac{t^{2 n+1}}{(2 n+1) !}
\end{aligned}
$$

which is precisely (6).

Finally, for $k=2 l+1, m=2 j+1$,

$$
\left[\frac{2^{-2 l} x^{2 l+1} t^{2 l+1}}{(1+a x)^{2 l+1}}\right]^{2 j+1} \frac{e^{t\left[\frac{1+b x}{1+a x}\right]}}{[(2 j+1)(2 l+1)] !}=\sum_{n=0}^{\infty} \mathcal{P}_{n}^{(a, b)}(x ; 2 l+1,2 j+1) \frac{t^{n}}{n !}
$$

Taking $t \rightarrow i t$,

$$
\left[\frac{2^{-2 l} x^{2 l+1}}{(1+a x)^{2 l+1}}\right]^{2 j+1} \frac{(i t)^{(2 l+1)(2 j+1)} e^{i t\left[\frac{1+b x}{1+a x}\right]}}{[(2 j+1)(2 l+1)] !}=\sum_{n=0}^{\infty} \mathcal{P}_{n}^{(a, b)}(x ; 2 l+1,2 j+1) \frac{(i t)^{n}}{n !}
$$

Thus,

$$
\begin{aligned}
& {\left[\frac{2^{-2 l} x^{2 l+1}}{(1+a x)^{2 l+1}}\right]^{2 j+1} \frac{\left(-t^{2}\right)^{(2 l j+l+j)}}{[(2 j+1)(2 l+1)] !}\left[-t \sin t\left(\frac{1+b x}{1+a x}\right)+i t \cos t\left(\frac{1+b x}{1+a x}\right)\right]} \\
& =\sum_{n=0}^{\infty}(-1)^{n} \mathcal{P}_{2 n}^{(a, b)}(x ; 2 l+1,2 j+1) \frac{t^{2 n}}{(2 n) !} \\
& \quad+i \sum_{n=0}^{\infty}(-1)^{n} \mathcal{P}_{2 n+1}^{(a, b)}(x ; 2 l+1,2 j+1) \frac{t^{2 n+1}}{(2 n+1) !} .
\end{aligned}
$$

Equating real and imaginary parts we get (7).

Since we obtain the unified Bernstein family in the case $a=0, b=-1$, we have the following corollary at once.

Corollary 13 For the unified Bernstein family, we have the following implicit summation formulae:

$$
\begin{aligned}
& \left(2^{1-2 l} x^{2 l}\right)^{m} \frac{\left(-t^{2}\right)^{l m}}{(2 l m) !} \cos t(1-x)=\sum_{n=0}^{\infty}(-1)^{n} \mathcal{B}_{2 n}(2 \operatorname{lm}, x) \frac{t^{2 n}}{(2 n) !} \\
& \left(2^{1-2 l} x^{2 l}\right)^{m} \frac{\left(-t^{2}\right)^{l m}}{(2 l m) !} \sin t(1-x)=\sum_{n=0}^{\infty}(-1)^{n} \mathcal{B}_{2 n+1}(2 \operatorname{lm}, x) \frac{t^{2 n+1}}{(2 n+1) !}
\end{aligned}
$$

and

$$
\begin{aligned}
& \left(2^{-2 l} x^{2 l+1}\right)^{2 j} \frac{\left(-t^{2}\right)^{(2 l+1) j}}{(2 j(2 l+1)) !} \cos t(1-x)=\sum_{n=0}^{\infty}(-1)^{n} \mathcal{B}_{2 n}((2 l+1)(2 j), x) \frac{t^{2 n}}{(2 n) !} \\
& \left(2^{-2 l} x^{2 l+1}\right)^{2 j} \frac{\left(-t^{2}\right)^{(2 l+1) j}}{(2 j(2 l+1)) !} \sin t(1-x)=\sum_{n=0}^{\infty}(-1)^{n} \mathcal{B}_{2 n+1}((2 l+1)(2 j), x) \frac{t^{2 n+1}}{(2 n+1) !}
\end{aligned}
$$


Finally,

$$
\begin{gathered}
{\left[2^{-2 l} x^{2 l+1}\right]^{2 j+1} \frac{\left(-t^{2}\right)^{(2 l j+l+j)}}{[(2 j+1)(2 l+1)] !} t \sin t(1-x)} \\
=\sum_{n=0}^{\infty}(-1)^{n} \mathcal{B}_{2 n}((2 l+1)(2 j+1), x) \frac{t^{2 n}}{(2 n) !} \\
{\left[2^{-2 l} x^{2 l+1}\right]^{2 j+1} \frac{\left(-t^{2}\right)^{(2 l j+l+j)}}{[(2 j+1)(2 l+1)] !} t \cos t(1-x)} \\
=\sum_{n=0}^{\infty}(-1)^{n} \mathcal{B}_{2 n+1}((2 l+1)(2 j+1), x) \frac{t^{2 n+1}}{(2 n+1) !} .
\end{gathered}
$$

On the other hand, taking $l=0$ in (8) and (9), we get the following relations for the Bernstein basis:

$$
\begin{aligned}
& x^{2 j} \frac{\left(-t^{2}\right)^{j}}{(2 j) !} \cos t(1-x)=\sum_{n=0}^{\infty}(-1)^{n} B_{2 j}^{2 n}(x) \frac{t^{2 n}}{(2 n) !}, \\
& x^{2 j} \frac{\left(-t^{2}\right)^{j}}{(2 j) !} \sin t(1-x)=\sum_{n=0}^{\infty}(-1)^{n} B_{2 j}^{2 n+1}(x) \frac{t^{2 n+1}}{(2 n+1) !}
\end{aligned}
$$

and

$$
\begin{aligned}
& x^{2 j+1} \frac{\left(-t^{2}\right)^{j}}{(2 j+1) !} t \sin t(1-x)=\sum_{n=0}^{\infty}(-1)^{n} B_{2 j+1}^{2 n}(x) \frac{t^{2 n}}{(2 n) !}, \\
& x^{2 j+1} \frac{\left(-t^{2}\right)^{j}}{(2 j+1) !} t \cos t(1-x)=\sum_{n=0}^{\infty}(-1)^{n} B_{2 j+1}^{2 n+1}(x) \frac{t^{2 n+1}}{(2 n+1) !} .
\end{aligned}
$$

Since the case $a=1, b=0$ gives the unified Bleimann-Butzer-Hahn family, we immediately obtain the following corollary.

Corollary 14 For the unified Bleimann-Butzer-Hahn family, we have the following implicit summation formulae:

$$
\begin{aligned}
& {\left[\frac{2^{1-2 l} x^{2 l}}{(1+x)^{2 l}}\right]^{m} \frac{\left(-t^{2}\right)^{l m}}{(2 l m) !} \cos \left(\frac{t}{1+x}\right)=\sum_{n=0}^{\infty}(-1)^{n} \mathcal{H}_{2 n}(2 \operatorname{lm}, x) \frac{t^{2 n}}{(2 n) !}} \\
& {\left[\frac{2^{1-2 l} x^{2 l}}{(1+x)^{2 l}}\right]^{m} \frac{\left(-t^{2}\right)^{l m}}{(2 l m) !} \sin \left(\frac{t}{1+x}\right)=\sum_{n=0}^{\infty}(-1)^{n} \mathcal{H}_{2 n+1}(2 \operatorname{lm}, x) \frac{t^{2 n+1}}{(2 n+1) !}}
\end{aligned}
$$

and

$$
\begin{aligned}
& {\left[\frac{2^{-2 l} x^{2 l+1}}{(1+x)^{2 l+1}}\right]^{2 j} \frac{\left(-t^{2}\right)^{(2 l+1) j}}{(2 j(2 l+1)) !} \cos \left(\frac{t}{1+x}\right)=\sum_{n=0}^{\infty}(-1)^{n} \mathcal{H}_{2 n}((2 l+1)(2 j), x) \frac{t^{2 n}}{(2 n) !},} \\
& {\left[\frac{2^{-2 l} x^{2 l+1}}{(1+x)^{2 l+1}}\right]^{2 j} \frac{\left(-t^{2}\right)^{(2 l+1) j}}{(2 j(2 l+1)) !} \sin \left(\frac{t}{1+x}\right)=\sum_{n=0}^{\infty}(-1)^{n} \mathcal{H}_{2 n+1}((2 l+1)(2 j), x) \frac{t^{2 n+1}}{(2 n+1) !} .}
\end{aligned}
$$


Finally,

$$
\begin{aligned}
& {\left[\frac{2^{-2 l} x^{2 l+1}}{(1+x)^{2 l+1}}\right]^{2 j+1} \frac{\left(-t^{2}\right)^{(2 l j+l+j)}}{[(2 j+1)(2 l+1)] !} t \sin \left(\frac{t}{1+x}\right)=\sum_{n=0}^{\infty}(-1)^{n} \mathcal{H}_{2 n}((2 l+1)(2 j+1), x) \frac{t^{2 n}}{(2 n) !}} \\
& {\left[\frac{2^{-2 l} x^{2 l+1}}{(1+x)^{2 l+1}}\right]^{2 j+1} \frac{\left(-t^{2}\right)^{(2 l j+l+j)}}{[(2 j+1)(2 l+1)] !} t \cos \left(\frac{t}{1+x}\right)} \\
& \quad=\sum_{n=0}^{\infty}(-1)^{n} \mathcal{H}_{2 n+1}((2 l+1)(2 j+1), x) \frac{t^{2 n+1}}{(2 n+1) !}
\end{aligned}
$$

Taking $l=0$ in (10) and (11), we get the following relations for the Bleimann-Butzer-Hahn basis:

$$
\begin{aligned}
& {\left[\frac{x}{1+x}\right]^{2 j} \frac{\left(-t^{2}\right)^{j}}{(2 j) !} \cos \left(\frac{t}{1+x}\right)=\sum_{n=0}^{\infty}(-1)^{n} H_{2 j}^{2 n}(x) \frac{t^{2 n}}{(2 n) !},} \\
& {\left[\frac{x}{1+x}\right]^{2 j} \frac{\left(-t^{2}\right)^{j}}{(2 j) !} \sin \left(\frac{t}{1+x}\right)=\sum_{n=0}^{\infty}(-1)^{n} H_{2 j}^{2 n+1} \frac{t^{2 n+1}}{(2 n+1) !} .}
\end{aligned}
$$

Finally,

$$
\begin{aligned}
& {\left[\frac{x}{1+x}\right]^{2 j+1} \frac{\left(-t^{2}\right)^{j}}{(2 j+1) !} t \sin \left(\frac{t}{1+x}\right)=\sum_{n=0}^{\infty}(-1)^{n} H_{2 j+1}^{2 n}(x) \frac{t^{2 n}}{(2 n) !}} \\
& {\left[\frac{x}{1+x}\right]^{2 j+1} \frac{\left(-t^{2}\right)^{j}}{(2 j+1) !} t \cos \left(\frac{t}{1+x}\right)=\sum_{n=0}^{\infty}(-1)^{n} H_{2 j+1}^{2 n+1}(x) \frac{t^{2 n+1}}{(2 n+1) !} .}
\end{aligned}
$$

Finally, we obtain a summation formula for the unified Bernstein and Bleimann-ButzerHahn basis as follows.

Theorem 15 For all $n, l \in \mathbb{N}_{0} ; a, b \in \mathbb{R}$, the following implicit summation formula holds true:

$$
\mathcal{P}_{n+l}^{(a, b)}(y ; k, m)=\sum_{p, r=0}^{l, n}\left(\begin{array}{l}
n \\
r
\end{array}\right)\left(\begin{array}{l}
l \\
p
\end{array}\right) \mathcal{P}_{n+l-r-p}^{(a, b)}(x ; k, m)\left[\frac{1+b y}{1+a y}-\frac{1+b x}{1+a x}\right]^{r+p} .
$$

Proof Letting $t \rightarrow t+u$ in (1) and then using the fact that

$$
\sum_{n=0}^{\infty} \sum_{l=0}^{\infty} A(l, n)=\sum_{n=0}^{\infty} \sum_{l=0}^{n} A(l, n-l)
$$

we get

$$
\begin{aligned}
{\left[\frac{2^{1-k} x^{k}(t+u)^{k}}{(1+a x)^{k}}\right]^{m} \frac{1}{(m k) !} e^{(t+u)\left[\frac{1+b x}{1+a x}\right]} } & =\sum_{n=0}^{\infty} \mathcal{P}_{n}^{(a, b)}(x ; k, m) \frac{(t+u)^{n}}{n !} \\
& =\sum_{n=0}^{\infty} \mathcal{P}_{n}^{(a, b)}(x ; k, m) \sum_{l=0}^{n} \frac{t^{n-l} u^{l}}{l !(n-l) !} \\
& =\sum_{n, l=0}^{\infty} \mathcal{P}_{n+l}^{(a, b)}(x ; k, m) \frac{t^{n} u^{l}}{n ! l !}
\end{aligned}
$$


and hence

$$
\left[\frac{2^{1-k} x^{k}(t+u)^{k}}{(1+a x)^{k}}\right]^{m} \frac{1}{(m k) !}=e^{-(t+u)\left[\frac{1+b x}{1+a x}\right]} \sum_{n, l=0}^{\infty} \mathcal{P}_{n+l}^{(a, b)}(x ; k, m) \frac{t^{n} u^{l}}{n ! l !}
$$

Multiplying both sides by $e^{(t+u)\left[\frac{1+b y}{1+a y}\right]}$ and then expanding the function $e^{(t+u)\left[\frac{1+b y}{1+a y}-\frac{1+b x}{1+a x}\right]}$, we get, after using (12) twice, that

$$
\begin{aligned}
& {\left[\frac{2^{1-k} x^{k}(t+u)^{k}}{(1+a x)^{k}}\right]^{m} \frac{1}{(m k) !} e^{(t+u)\left[\frac{1+b y}{1+a y}\right]}} \\
& =e^{(t+u)\left[\frac{1+b y}{1+a y}-\frac{1+b x}{1+a x}\right]} \sum_{n, l=0}^{\infty} \mathcal{P}_{n+l}^{(a, b)}(x ; k, m) \frac{t^{n} u^{l}}{n ! l !} \\
& \quad=\sum_{n, l=0}^{\infty} \sum_{r=0}^{\infty} \mathcal{P}_{n+l}^{(a, b)}(x ; k, m) \frac{\left[\frac{1+b y}{1+a y}-\frac{1+b x}{1+a x}\right]^{r}}{r !}(t+u)^{r} \frac{t^{n} u^{l}}{n ! l !} \\
& =\sum_{n, l, p, r=0}^{\infty} \mathcal{P}_{n+l}^{(a, b)}(x ; k, m)\left[\frac{1+b y}{1+a y}-\frac{1+b x}{1+a x}\right]^{r+p} \frac{t^{n+r} u^{p+l}}{n ! l ! r ! p !} .
\end{aligned}
$$

Now, using (12) with the index pairs $(n, r)$ and $(l, p)$, we get

$$
\begin{aligned}
& {\left[\frac{2^{1-k} x^{k}(t+u)^{k}}{(1+a x)^{k}}\right]^{m} \frac{1}{(m k) !} e^{(t+u)\left[\frac{1+b y}{1+a y}\right]}} \\
& \quad=\sum_{n, l=0}^{\infty} \sum_{p, r=0}^{l, n}\left(\begin{array}{l}
n \\
r
\end{array}\right)\left(\begin{array}{l}
l \\
p
\end{array}\right) \mathcal{P}_{n+l-r-p}^{(a, b)}(x ; k, m)\left[\frac{1+b y}{1+a y}-\frac{1+b x}{1+a x}\right]^{r+p} \frac{t^{n} u^{l}}{n ! l !} .
\end{aligned}
$$

Since the left-hand side is equal by (13) to

$$
\left[\frac{2^{1-k} x^{k}(t+u)^{k}}{(1+a x)^{k}}\right]^{m} \frac{1}{(m k) !} e^{(t+u)\left[\frac{1+b y}{1+a y}\right]}=\sum_{n, l=0}^{\infty} \mathcal{P}_{n+l}^{(a, b)}(y ; k, m) \frac{t^{n} u^{l}}{n ! l !}
$$

the proof is completed by comparing the coefficients of $\frac{t^{n} u^{l}}{n ! l !}$ in (14) and (15).

In the case $a=0, b=-1$, we obtain the following result for the unified Bernstein family at once.

Corollary 16 For all $n, l \in \mathbb{N}_{0}$, the following implicit summation formula:

$$
\mathcal{B}_{n+l}(m k, y)=\sum_{p, r=0}^{l, n}\left(\begin{array}{l}
n \\
r
\end{array}\right)\left(\begin{array}{l}
l \\
p
\end{array}\right) \mathcal{B}_{n+l-r-p}(m k, x)[x-y]^{r+p}
$$

holds true for the unified Bernstein family. Taking $k=1$ in (16), we get the following relation for the Bernstein basis:

$$
B_{m}^{n+l}(y)=\sum_{p, r=0}^{l, n}\left(\begin{array}{l}
n \\
r
\end{array}\right)\left(\begin{array}{l}
l \\
p
\end{array}\right) B_{m}^{n+l-r-p}(x)[x-y]^{r+p} .
$$


Since the case $a=1, b=0$ gives the unified Bleimann-Butzer-Hahn family, we have the following result.

Corollary 17 For all $n, l \in \mathbb{N}_{0}$, the following implicit summation formula:

$$
\mathcal{H}_{n+l}(m k, y)=\sum_{p, r=0}^{l, n}\left(\begin{array}{l}
n \\
r
\end{array}\right)\left(\begin{array}{l}
l \\
p
\end{array}\right) \mathcal{H}_{n+l-r-p}(m k, x)[x-y]^{r+p}
$$

holds true for the unified Bleimann-Butzer-Hahn family. Upon taking $k=1$ in (17), we get the following relation for the Bleimann-Butzer-Hahn basis:

$$
H_{m}^{n+l}(y)=\sum_{p, r=0}^{l, n}\left(\begin{array}{l}
n \\
r
\end{array}\right)\left(\begin{array}{l}
l \\
p
\end{array}\right) H_{m}^{n+l-r-p}(x)[x-y]^{r+p}
$$

\section{Competing interests}

The authors declare that they have no competing interests.

\section{Authors' contributions}

All authors completed the paper together. All authors read and approved the final manuscript.

\section{Acknowledgements}

Dedicated to Professor Hari M Srivastava.

Received: 30 November 2012 Accepted: 31 January 2013 Published: 13 March 2013

\section{References}

1. Simsek, Y: Constructing a new generating function of Bernstein type polynomials. Appl. Math. Comput. 218, 1072-1076 (2011)

2. Acikgoz, M, Aracı, S: On generating function of the Bernstein polynomials. Proceedings of the International Conference on Numerical Analysis and Applied Mathematics. AlP Conf. Proc. 1281, 1141-1143 (2010)

3. Bayad, A, Kim, T: Identities involving values of Bernstein, $q$-Bernoulli, and $q$-Euler polynomials. Russ. J. Math. Phys. 18(2), 133-143 (2011)

4. Bernstein, SN: Démonstration du théorème de Weierstrass fondée sur la calcul des probabilités. Commun. Soc. Math. Kharkov 13, 1-2 (1912-13)

5. Bleimann, G, Butzer, PL, Hahn, L: A Bernstein-type operator approximating continuous functions on the semi-axis Indag. Math. 42, 255-262 (1980)

6. Busé, L, Goldman, R: Division algorithms for Bernstein polynomials. Comput. Aided Geom. Des. 25, 850-865 (2008)

7. Kim, M-S, Kim, T, Lee, B, Ryoo, C-S: Some identities of Bernoulli numbers and polynomials associated with Bernstein polynomials. Adv. Differ. Equ. 2010, Article ID 305018 (2010)

8. Kim, T, Jang, L-J, Yi, H: A note on the modified q-Bernstein polynomials. Discrete Dyn. Nat. Soc. (2010). doi:10.1155/2010/706483

9. Morin, G, Goldman, R: On the smooth convergence of subdivision and degree elevation for Bézier curves. Comput. Aided Geom. Des. 18, 657-666 (2001)

10. Phillips, GM: Interpolation and Approximation by Polynomials. CMS Books in Mathematics/Ouvrages de Mathématiques de la SMC, vol. 14. Springer, New York (2003)

11. Simsek, Y, Acikgoz, M: A new generating function of (q-) Bernstein-type polynomials and their interpolation function. Abstr. Appl. Anal. 2010, Article ID 769095 (2010)

12. Zorlu, S, Aktuglu, H, Ozarslan, MA: An estimation to the solution of an initial value problem via $q$-Bernstein polynomials. J. Comput. Anal. Appl. 12,637-645 (2010)

13. Ulrich, A, Mircea, I: The Bleimann-Butzer-Hahn operators old and new results. Appl. Anal. 90(3-4), 483-491 (2011)

14. Ozarslan, MA: Unified Apostol-Bernoulli, Euler and Genocchi polynomials. Comput. Math. Appl. 62(6), 2452-2462 (2011)

15. Luo, Q-M, Srivastava, HM: Some generalizations of the Apostol-Bernoulli and Apostol-Euler polynomials. J. Math. Anal. Appl. 308(1), 290-302 (2005)

16. Luo, Q-M: On the Apostol-Bernoulli polynomials. Cent. Eur. J. Math. 2(4), 509-515 (2004)

17. Luo, Q-M, Srivastava, HM: Some relationships between the Apostol-Bernoulli and Apostol-Euler polynomials. Comput. Math. Appl. 51(3-4), 631-642 (2006)

18. Srivastava, HM: Some formulas for the Bernoulli and Euler polynomials at rational arguments. Math. Proc. Camb. Philos. Soc. 129(1), 77-84 (2000)

19. Luo, Q-M: Fourier expansions and integral representations for the Genocchi polynomials. J. Integer Seq. 12, Article ID 09.1.4 (2009) 
20. Luo, Q-M: Extension for the Genocchi polynomials and its Fourier expansions and integral representations. Osaka J. Math. 48(2), 291-309 (2011)

21. Luo, Q-M: Apostol-Euler polynomials of higher order and Gaussian hypergeometric functions. Taiwan. J. Math. 10, 917-925 (2006)

22. Srivastava, HM, Choi, J: Series Associated with the Zeta and Related Functions. Kluwer Academic, Dordrecht (2001)

doi:10.1186/1687-1847-2013-55

Cite this article as: Özarslan and Bozer: Unified Bernstein and Bleimann-Butzer-Hahn basis and its properties. Advances in Difference Equations 2013 2013:55.

Submit your manuscript to a SpringerOpen ${ }^{\odot}$ journal and benefit from:

- Convenient online submission

Rigorous peer review

- Immediate publication on acceptance

- Open access: articles freely available online

- High visibility within the field

- Retaining the copyright to your article

Submit your next manuscript at $\gg$ springeropen.com 